\title{
Operation nach Strayer-Thom
}

\author{
Dieter Herold, Johann Kusch, Stephan Martin, Andreas Tenger, Christian H. Siebert
}

\section{Zusammenfassung}

Die Operationsmethode nach StrayerThom stellt ein geeignetes Verfahren zur Korrektur des strukturellen Spitzfußes bei der infantilen Zerebralparese mit geringem Risiko der muskulären Insuffizienz dar. Dabei wird selektiv der M. gastrocnemius alternativ zur üblichen Achillotenotomie verlängert. Die beschriebene Technik ist einfach und ohne große Risiken durchzuführen. Es können gute kosmetische Ergebnisse ohne sichtbare subkutane „Muldenbildung“ im Wadenbereich erreicht werden.

\section{Operations According to Strayer-Thom}

The surgical technique according to Strayer-Thom represents a suitable method for the correction of the structural pes equinus in cases of infantile cerebral paresis and carries only a low risk of muscular insufficiency. In this procedure the gastrocnemius muscle is lengthened as an alternative to the usual achillotenotomy. The described technique is simple and can be performed without major risks. Good cosmetic results can be achieved without the formation of subcutaneous depressions in the sural region.

\section{Einleitung}

Der Spitzfuß stellt in der orthopädischen Betreuung der Patienten mit zerebralen Bewegungsstörungen ein regelmäßiges, wenn nicht sogar das häufigste Problem bei gehfähigen Patienten dar.

Als Folge des spitzfüßigen Gehens und Stehens stellen sich im Wachstum Formveränderungen der Gelenke und der Fußform ein.

Die fehlende Fersenbelastung mündet in eine verminderte Größenentwicklung des Kalkaneus mit resultierender Störung der Fußstatik im Wachstum sowie verminderter Belastungsfähigkeit. Die typische Dreiecksform der Füße ist das Resultat dieser lang anhaltenden Minderbelastung der Ferse. Gleichzeitig führt die vermehrte Belastung des Vorfußes zur Spreizfußdeformität, wodurch es aufgrund der ungünstigen Fußstatik zur verminderten Belastungsfähigkeit kommt.

OP-JOURNAL 2007; 23: 160-163

(c) Georg Thieme Verlag KG Stuttgart • New York
Ursache des Spitzfußes ist bei der zerebralen Bewegungsstörung in der Mehrheit eine Tonuserhöhung der Wadenmuskulatur. Aufgrund der pathologischen Reflexe führt die Dorsalextension im oberen Sprunggelenk zu einer vermehrten Aktivität der Wadenmuskulatur mit resultierender Spitzfußstellung. Im Rahmen der klinischen Untersuchung muss differenziert werden, welche Muskelanteile der Wade für die Spitzfußstellung auslösend sind. Mit dem Test nach Silverskiöld kann durch Prüfen der Beweglichkeit des Sprunggelenkes in Kniebeugung und Kniestreckung eine Differenzierung der Spastik des M. trizeps erfolgen (Abb.1).

Differenziert werden muss im Rahmen der klinischen Untersuchung ebenfalls zwischen strukturell bedingten Spitzfußstellungen und den aktivitätsbedingten dynamischen Spitzfußstellungen.

Auf die Möglichkeiten der konservativen Behandlung der Fehlstellung mittels physiotherapeutischer Maßnahmen und Orthesen soll in diesem Beitrag nicht näher eingegangen werden.
In der Literatur werden zahlreiche operative Maßnahmen zur Korrektur der Fehlstellung angegeben. Grundsätzlich lassen sich diese Verfahren in 3 Kategorien einteilen:

1. Minderung der Wadenspannung durch Beeinflussung der Innervation (Stoffel-Operation) [4]

2. Spannungsminderung durch Eingriffe am M. gastrocnemius/soleus [3, 5, 6]

3. Verlängerung der Achillessehne.

Ziel aller Verfahren ist die Minderung der Spitzfußstellung. Bei der Auswahl des Operationsverfahrens ist die Erkennung der Ursache der Spitzfußstellung Basis der Therapie.

Die Unterscheidung der Fehlstellungen in „dynamische und kontrakte Spitzfüße“ ist für die weitere Therapieentscheidung grundlegend. Ziel der Therapie ist es, eine belastungsfähige Ferse für das Stehen und Gehen zu erzielen, sowie Pathologien der Sprunggelenke und der Fußstatik zu vermeiden.

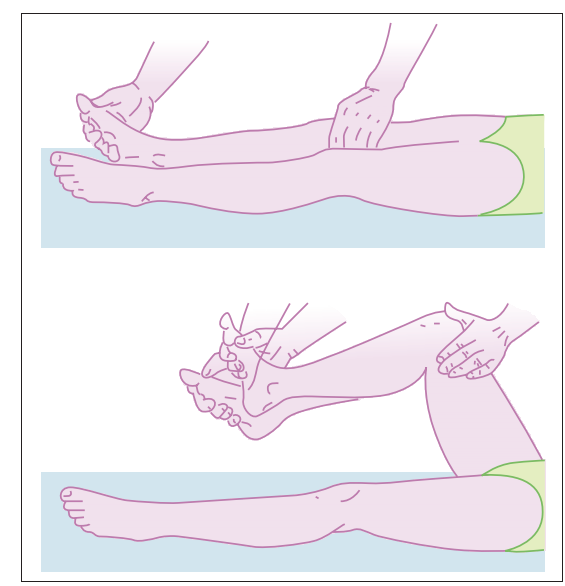

Abb. 1 Test nach Silverskiöld. Positives „Transmissionsphänomen“: Bei Kniebeugung bessert sich die Spitzfußkontraktur. Der M. gastrocnemius ist stärker verkürzt als der $\mathrm{M}$. soleus. 
Auf die Problematik des operativen Vorgehens hatte bereits Bleck hingewiesen [1]:

"What surgical procedure do you perform to correct an equinus deformity? Many have been proposed: neurectomy of branches of tibial nerve to the gastrocnemius and/or soleus muscles, gastrocnemius lengthening, gastrocnemius origin recession, Achilles tendon lengthening and Achilles tendon translocation. All are designed to reduce the increased stretch reflex and to lengthen the muscle. All weaken the muscle, which one strikes the best compromise between relieving the contracture and preserving the strength? Lengthening weakens the muscle. We have no solution except to try to avoid too much lengthening of the tendon."

Bei der Spitzfußstellung aufgrund einer Spastik der Wadenmuskulatur führt die Verlängerung der Achillessehne (gleich welcher Technik) zu einer verminderten Vorspannung der gesamten Trizepsmuskulatur und damit zu einer Reduktion der spastikbedingten Plantarflexion des Fußes. Nachteilig bei diesem Vorgehen ist die unvermeidbare Kraftminderung der Wadenmuskulatur durch die verminderte Vorspannung des M. triceps. Langfristig bestehen erhebliche Risiken der Entwicklung eines Hackenfußes als Folge des geänderten Muskelgleichgewichtes am Sprunggelenk [2].

Eine proximale Verlängerung des $\mathrm{M}$. gastrocnemius durch Versetzung der Ursprüngsköpfe des $M$. gastrocnemius wurde durch Silverskiöld beschrieben. Es zeigte sich jedoch, dass die proximale Verlängerung sich günstig zur Verminderung einer Kniebeugekontraktur auswirkt, die Valgusstellung des Kalkaneus jedoch weniger korrigiert wird.

Verfahren zur distalen Verlängerung des M. gastrocnemius wurden bereits Anfang des letzten Jahrhunderts beschrieben. Bereits 1913 wurde daher die selektive Operation des M. gastrocnemius durch Vulpius [6] beschrieben. Ein ähnliches Vorgehen schilderte Strayer 1950 [5].

Im Folgenden soll die intramuskuläre Verlängerung in der Modifikation von Strayer und Thom dargestellt und erläutert werden.

\section{Vorteile}

- Korrektur des strukturellen Spitzfußes bei der ICP mit geringem Risiko der muskulären Insuffizienz durch selektive Verlängerung des M. gastrocnemius ohne Verlängerung des $\mathrm{M}$. soleus

- Gute kosmetische Ergebnisse ohne sichtbare subkutane „Muldenbildung“ im Wadenbereich.

\section{Nachteile}

- Bei Kindern im Wachstumsalter besteht grundsätzlich Rezidivgefahr, da Narben das Längenwachstum des Muskels beeinträchtigen können.

- Über einen Zeitraum von 3-6 Monaten müssen Lauforthesen und Nachtlagerungsschienen getragen werden.

Indikation

- Struktureller Spitzfuß bei der ICP mit positivem „Transmissionsphänomen“ (M. gastrocnemius stärker verkürzt als M. soleus)

- Sowohl als einzelner Eingriff als auch im Rahmen eines „Mehretageneingriffes"

- Ausgeprägte Aktionsspastik bei forcierter Dorsalextension im Fußgelenk.

\section{Kontraindikation}

- Verkürzung des gesamten M. triceps surae

- Kapsuläre Spitzfußkontraktur

- Geringe nicht persistierende Muskelverkürzung, die durch konservative Behandlung korrigierbar wären.

\section{Operationsplanung}

- Röntgenaufnahme des gesamten Fußes in 2 Ebenen

- Blutsperre nicht zwingend erforderlich

- Desinfektion und steriles Abdecken mit beweglichem Knie- und Fußgelenk

- Kontrolle der Durchblutung.

\section{Instrumentarium}

- Grundsieb Fußinstrumentarium mit Wundspreizer.

\section{Anästhesie und Lagerung}

- Der Eingriff wird in Bauchlage mit Unterlagerung des Thorax in Intubationsnarkose durchgeführt, evtl. zusätzlich Sakralanästhesie.

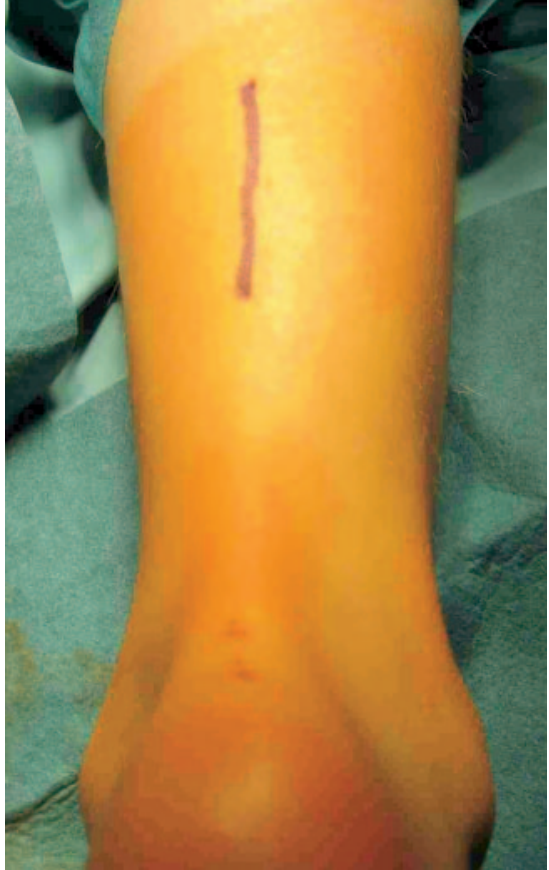

Abb. 2 Hautschnitt.

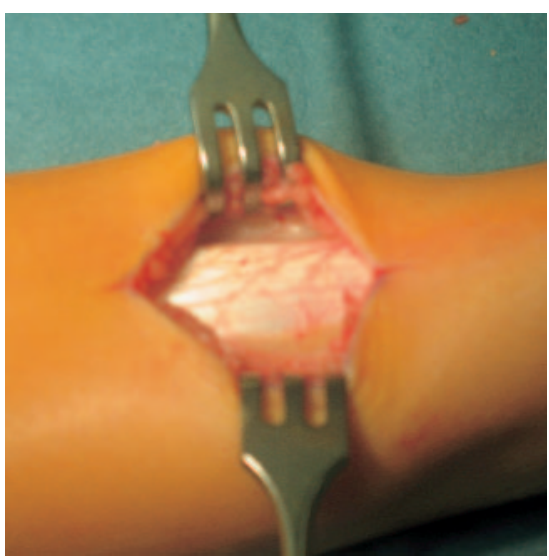

Abb. 3 Darstellen der Faszie des M. gastrocnemius.

\section{OP-Technik}

1. Zugang: Ca. $3 \mathrm{~cm}$ langer Schnitt proximal der Achillessehne in der Mitte des Unterschenkels. Die V. saphena parva wird dargestellt und möglichst schonend präpariert (Abb. 2).

2. Darstellung der Faszie des M. gastrocnemius (Abb. 3)

3. Aufsuchen der „Umschlagfalte“ der Faszie und Lösen der Gastrocnemiusvon der Soleusfaszie mit dem Overholt. In einigen Fällen kann ein Lösen in 2-3 Schritten erforderlich sein (Abb. 4).

4. Durchtrennen der Gastrocnemiusfaszie möglichst weit distal (Abb.5) 


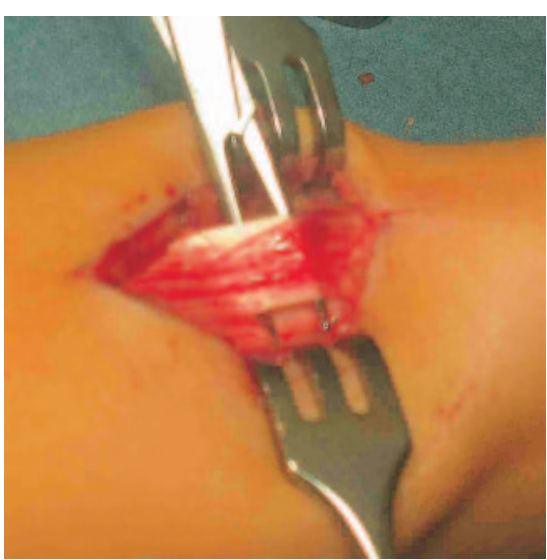

Abb.4 Spalten der Faszienblätter des M. gastrocnemius und $\mathrm{M}$. soleus.

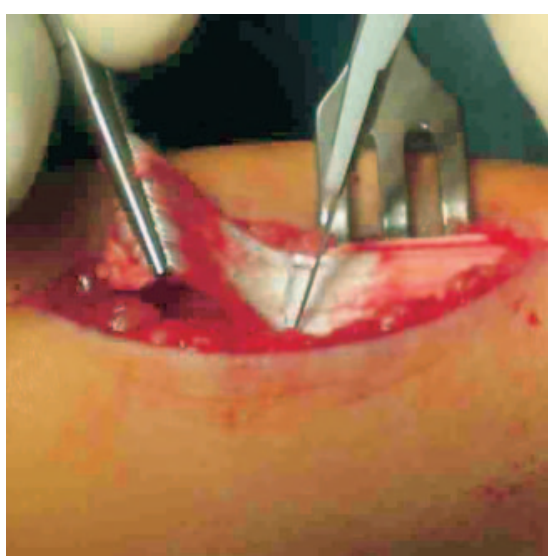

Abb. 6 Präparation des oberflächigen Blattes nach proximal.

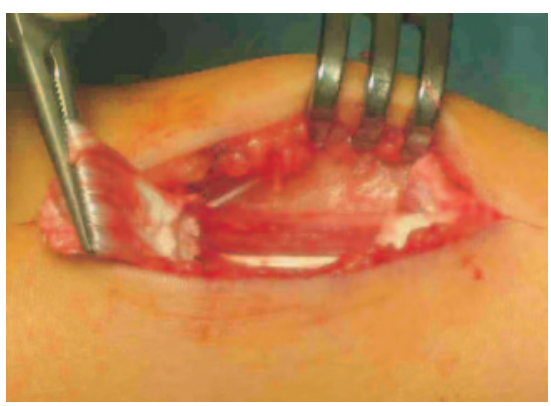

Abb. 8 Durchtrennung von tiefen, sagittal verlaufenden Septen, ggf. auch Sehne der M. plantaris.

5. Greifen der gelösten Faszie mit einem Klemmchen und Lösen der beiden Faszienblätter über $3-5 \mathrm{~cm}$ nach proximal. Dieses erfolgt je nach Anatomie stumpf oder durch „schabendes“ Schneiden mit dem Skalpell und wird erleichtert durch die Dorsalextension des OSG. Die zu lösenden Faszien „verschieben“ sich dann durch das OP-Feld (Abb. 6).

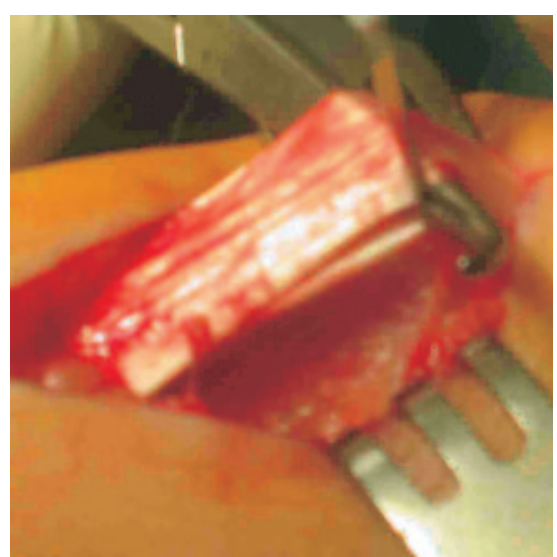

Abb. 5 Ablösen des oberflächigen Blattes distal.

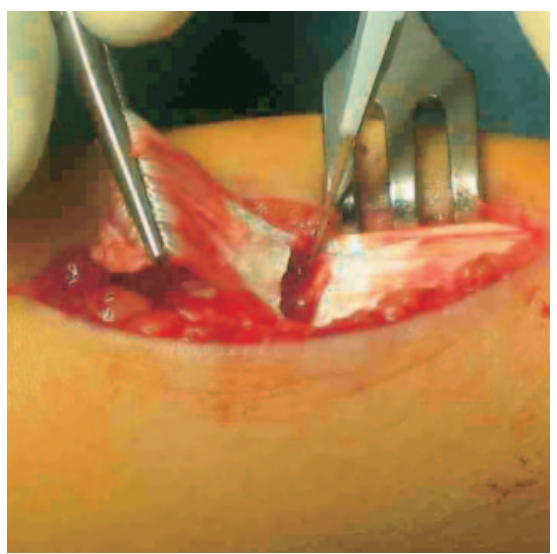

Abb. 7 Quere Durchtrennung des tiefen Blattes proximal.

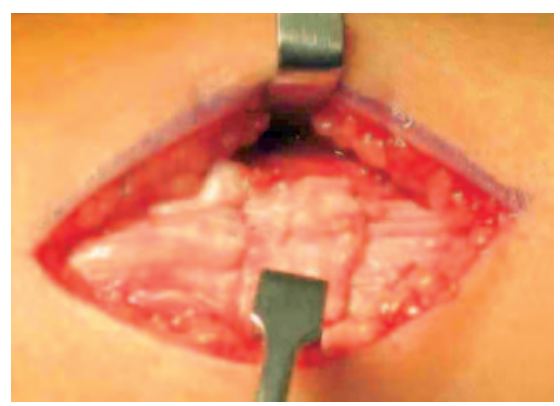

Abb. 9 End-zu-End-Naht der Blätter bei orthograder Fußstellung.

6. Quere Durchtrennung der Soleusfaszie unter Schonung des M. soleus (Abb. 7)

7. Bei inkompletter Korrekturmöglichkeit ist es erforderlich, zusätzlich tiefe sagittal verlaufende Septen des M. gastrocnemius zu inzidieren (Abb. 8).

8. Z-förmige Adaptation der beiden Faszienblätter in Neutralnullstellung unter leichter Spannung mit einzelnen
„U“-Nähten (z. B. PDS, Fa. Ethicon). Es handelt sich um Situationsnähte, da die Kontinuität des M. soleus und der Achillessehne nicht unterbrochen wurde ist die Spannung auf der Naht sehr gering. Schichtweiser Wundverschluss. Auf eine Drainage kann verzichtet werden (Abb. 9).

\section{Nachbehandlung}

- Verbandwechsel und Wundkontrollen erfolgen in dreitägigen Abständen.

- Das Nahtmaterial wird am 12. postoperativen Tag entfernt.

- Für 2 Wochen wird ein Oberschenkelgips angelegt. Dann Wechsel auf eine Unterschenkelgips für weitere 4 Wochen nach vorheriger Entnahme eines Abdrucks für US-Lagerungs- und Funktionsorthesen.

- Nach Gipsabnahme wird mit individueller Bewegungstherapie begonnen.

- Die zwischenzeitlich gefertigten Orthesen werden angepasst.

Fehlermöglichkeiten und

\section{Komplikationen}

- Falsche Indikationsstellung bei nicht vorhandenem Transmissionsphänomen, wie z.B. bei Hemispastik oder anderen neuromuskuären Grunderkrankungen, wie Muskeldystrophie o. ä.

- Fehlplatzierung des Hautschnittes

- Verletzung der Vena saphena parva oder des N. saphenus parvus.

\section{Ergebnisse}

In unserer Abteilung werden jährlich ca. 40 Operationen nach Strayer-Thom durchgeführt, davon viele beidseits.

Hierbei handelt sich dabei um Patienten, die regelmäßig prä- und postoperativ in unserer neuroorthopädischen Ambulanz fachärztlich betreut werden.

Wir können auf durchweg gute Ergebnisse mit weniger als einer Rezidivoperation pro Jahr zurückblicken.

Überkorrekturen oder Insuffizienzen wurden bisher nicht beobachtet. 


\section{Literatur}

${ }^{1}$ Bleck EE. Orthopaedic management in cerebral palsy. London: MacKeith Press, 1987: $52-54$

2 Segal LS, Thomas SE, Mazur JM, Mauterer M. Calcaneal gait in spastic diplegia after heel cord lengthening: a study with gait analysis. J Pediatr Orthop 1989; 9: 697-701

3 Silver CM, Simon SD. Gastrocnemius muscle recession (Silverskiöld operation) for spastic equinus deformity in cerebral palsy. J Bone Joint Surg [Am] 1959; 41: 1021 - 1028

4 Stoffel A. The treatment of spastic contracture. Am J Orthop Surg 1913; 10: 611 -644

${ }^{5}$ Strayer LM. Recession of the gastrocnemius: An operation to relieve spastic contracture of the calf muscles. J Bone Joint Surg [Am] 1950; 32: 671

${ }^{6}$ Vulpius O. Orthopädische Operationslehre. 3. Aufl. Stuttgart: Ferdinand Enke, 1924

\section{Dr. med. Dieter Herold}

Arzt für Orthopädie, Kinderorthopädie, Abteilungsleiter Kinder- und Neuro-

orthopädie

\section{Dr. med. Johann Kusch}

Leitender Oberarzt Kinder- und

Neuroorthopädie

Dr. med. Stephan Martin

Sektionsleiter Neuroorthopädie

Dr. med. Andreas Tenger

Sektionsleiter Neuroorthopädie

Prof. Dr. med. Christian H. Siebert

Leitender Arzt

Klinik 1

Diakoniekrankenhaus Annastift e.V.

Anna-von-Borries-Straße 1 - 7

30625 Hannover 